\title{
An sEMG-based Interface to give People with Severe Muscular Atrophy control over Assistive Devices
}

\author{
Jörn Vogel and Annette Hagengruber
}

\begin{abstract}
Injuries, accidents, strokes, and other diseases can significantly degrade the capabilities to perform even the most simple activities in daily life. While assistive technology becomes more and more available to the people affected, there is still a big need for user interfaces suitable for people without functional hand movement. A large share of these cases involves neuromuscular diseases, which lead to severely reduced muscle function. However, even though affected people are no longer able to functionally move their limbs, residual muscle function can still be existent. Previous work has shown that this residual muscular activity can suffice, to create an EMG-based user interface, and e.g. allow for control of assistive devices. In this paper, we enhance this user interface with additional EMG-features and an improved training paradigm in order to increase information extraction from recordings of strongly atrophic muscles. The interface was tested and validated by subjects with severe spinal muscular atrophy. Results show that the used methods improve the decoding and thereby allow for a considerable increase in performance when controlling a robotic manipulator in a $3 D$ reaching task.
\end{abstract}

\section{INTRODUCTION}

In our daily life, activities such as drinking, eating, or taking a walk in the park are elementary parts that we perform without thinking. However, neuro-muscular diseases, trauma or stroke can result in paralysis and thereby strongly inhibit one's ability to perform these tasks, which reduces the mobility and independence of the person affected. In severe cases, even simple activities of daily living, and thus, a selfdetermined life in one's own home may become impossible, and people require personal care around-the-clock.

For some time past, assistive technologies for people with motor disabilities have been developed to provide help and relief in daily life. For one, power wheelchairs can, to a large extent, restore the mobility of the individual. Furthermore, technical aids are developed to restore the manipulation capabilities of people with upper limb paralysis. While at first passive and active arm support systems, were built to provide help for people with remaining but weak arm and hand function [1], [2], nowadays, robotic manipulators for people without remaining hand function or arm movement become more and more available [3].

However, control over the aforementioned manipulators is usually achieved with a joystick, and therefore requires the user to have remaining functionality in hand and finger

This work was supported by the Bavarian Ministry of Economic Affairs, Energy and Technology (StMWi) by means of the projects Smart-Assist and SMiLE

The authors are with the Institute of Robotics and Mechatronics, German Aerospace Center (DLR), Oberpfaffenhofen/Germany.

Contact: joern.vogel (at) dlr.de movement [4]. This requirement prevents many people with paralysis from efficient use of the already available assistive technology. Many alternative approaches have been investigated, e.g. the Tongue Drive [?], which consists of touch sensors fixed on the palate to be activated with the tip of the tongue, or the measurement of eyebrow movements via piezo-based sensors [?].

Another solution to this problem is the use of BrainComputer Interfaces (BCI). In the past years, invasive interfaces have been demonstrated to provide people with tetraplegia with continuous control over robotic manipulators [5], [6]. The high spatial and temporal resolution of these implanted BCIs allows for a natural control scheme, in which motor imagination from one limb is mapped to motion of a robotic arm. In comparison, non-invasive BCIs provide a much lower signal bandwidth and therefore are typically used to decode discrete control commands or operate in a less natural control scheme [7], [8]. One alternative approach to BCIs can be the use of surface Electromyography sEMG as a source for a human machine interface HMI. Currently, EMGbased HMIs are commonly used for control of myoelectric hand prosthesis. In [9] it was shown, that sEMG recordings from residual muscle fibers of people with severe muscular atrophy can be used to continuously control the endeffector of a simulated robotic system. In this work, we present an enhanced version of such an sEMG-based HMI, and assert its functionality in a $2 \mathrm{D}$ and $3 \mathrm{D}$ reach task.

\section{INTERFACE DESIGN}

Joysticks, which are typically used to interface assistive manipulators, provide the user with a continuous 3 degree of freedom (DoF) control input. This control signal is then mapped to command the endeffector velocity of the robot, either in translational or rotational space. Naturally, a 3DoF interface also allows for the control of lower dimensional devices like a wheelchair as well as additional functions like seat configuration, or e.g. a cursor on a tablet computer.

However, joysticks are not suitable for people with a severe muscular atrophy resulting in loss of hand functionality. Thus, our goal is to provide people with severe muscular atrophy with such a continuous $3 \mathrm{DoF}$ interface, based on the measurement of their remaining muscular activity. Such an interface can then be used to control the endeffector velocity of a robotic device. In [9] a proof of concept study was conducted, in which two users with spinal muscular atrophy (SMA) were able to control the translational movement of a simulated robotic system. In that study, an amplitude based 

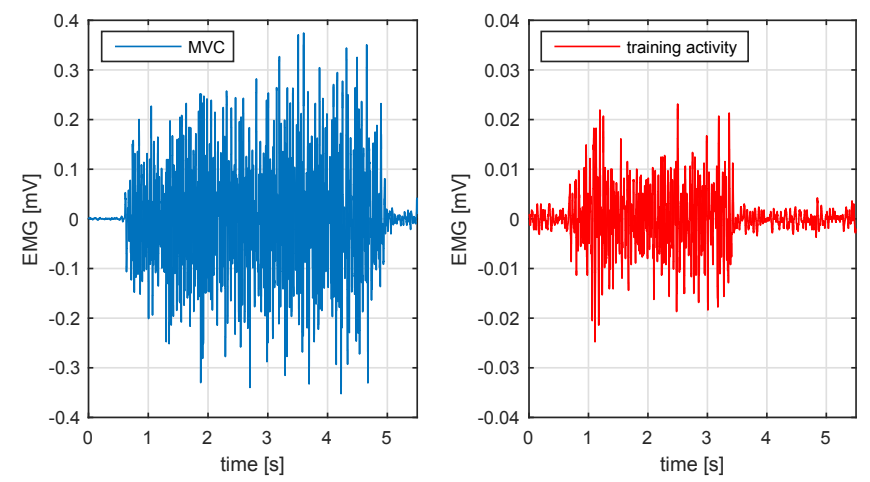

Fig. 1. Left: Exemplary sEMG-signal at maximum voluntary contraction. Right: sEMG activity recorded from the same electrode during usage of an sEMG-based interface, which is an order of magnitude lower in amplitude.

representation of the sEMG signals was used in combination with a neural-network to decode the control signals. Since sEMG measurements of muscular activity are composted of the superposition of multiple motor unit action potentials (MUAP), much information is encoded in the signals amplitude (see Figure 1).

However, analysis of sEMG-recordings from people with progressed spinal muscular atrophy reveals, that variation in amplitude may not be as distinct as in non-pathological sEMG-signals. Figure 2 shows an sEMG-recording from a person with SMA at maximum voluntary contraction (MVC). Compared to the non-pathological MVC signal, the amplitude is an order of magnitude lower. Zooming in on the signal reveals, that the signal is composed of a sequence of motor unit action potentials and as such, reduced activity will not result in a prominent change in amplitude, but rather in a change of rate of MUAPs.

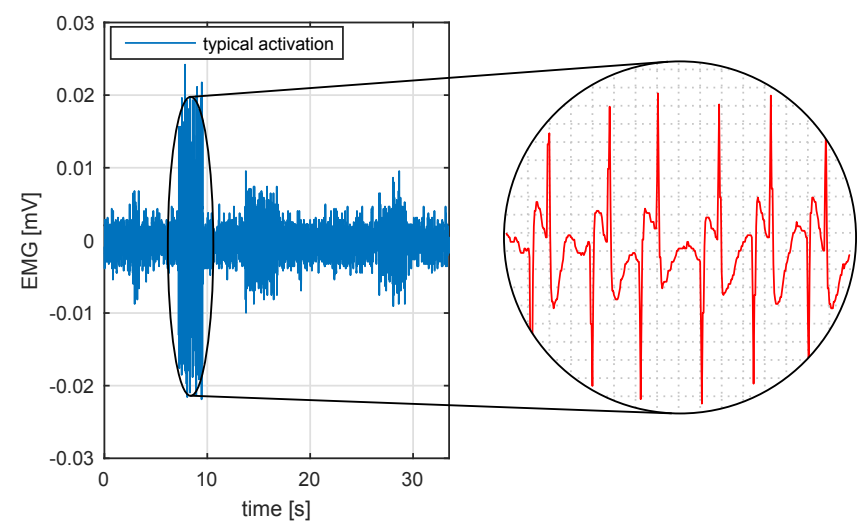

Fig. 2. EMG recording of maximum voluntary contraction from an atrophic muscle. In the detailed view on the right single MUAP recordings are visible.

\section{A. EMG decoding}

In order to effectively acquire information from this kind of sEMG-signals, additional features, representing time and frequency-based components, should be applied. Thus, in this work we extended the decoding procedure to use the following four features:

- sEMG-amplitude
- Slope-Sign-Change

- Zero-Crossing-Rate

- Waveform-Length

To acquire the amplitude of the sEMG-signal, the polynomial low-pass filter as presented in [9] is used. The other features are calculated according to Eqs. (1), (2) and (3), as introduced in [10]:

Slope-Sign-Change:

$$
\begin{aligned}
& R_{\mathrm{ssc}}=\sum_{i=2}^{N-1} f\left[\left(x_{i}-x_{i-1}\right)\left(x_{i}-x_{i+1}\right)\right] \\
& f(\gamma)= \begin{cases}1 & \text { if } \gamma>\mu_{\mathrm{ssc}} \\
0 & \text { otherwise }\end{cases}
\end{aligned}
$$

Zero-Crossing-Rate:

$$
\begin{array}{r}
R_{\mathrm{zc}}=\sum_{i=1}^{N-1}\left[\operatorname{sign}\left(-x_{i} x_{i+1}\right) g\left(\operatorname{abs}\left(x_{i}-x_{i+1}\right)\right)\right] \\
g(\gamma)= \begin{cases}1 & \text { if } \gamma>\mu_{\mathrm{zc}} \\
0 & \text { otherwise }\end{cases}
\end{array}
$$

Waveform-Length:

$$
R_{\mathrm{wl}}=\sum_{i=1}^{N-1}\left[a b s\left(x_{i+1}-x_{i}\right)\right]
$$

All used features are calculated online over a window of $N=150$ samples $(x)$ on each sEMG-channel. The features $R_{\mathrm{ssc}}$ and $R_{\mathrm{zc}}$ allow for adjustment to baseline noise using the parameters $\mu_{\mathrm{ssc}}$ and $\mu_{\mathrm{zc}}$, respectively. These parameters can also effectively be used, in order to implement an activity detection, which on the one hand allows the user to create an output signal with zero velocity, on the other hand, this activity detection can be used to coordinate the procedure of recording training data for the decoding algorithm.

\section{B. Decoding Procedure}

Based on the four features, as described above, sEMG decoding is acquired using Gaussian Process regression. The procedure for setting up this decoder consists of the following steps:

- Application of electrodes,

- Recording of rest signal,

- Configuration of features,

- Recording of training data,

- Calculation of training dataset,

- Gaussian process modeling,

- Online regression.

1) Application of electrodes: sEMG-signals are recorded using eight Delsys Trigno wireless sEMG-sensors. These sensors are attached to the skin surface using medical grade double-sided adhesive tape and signals are wirelessly transferred to the Trigno base-station and digitized at a sampling rate of $1 \mathrm{kHz}$. 
2) Recording of rest signal: Once the electrodes are placed, 5 seconds of sEMG-data $X_{\text {rest }}^{8 \times 5000}$ are recorded, while the user is in a resting state. This data is used to calculate the bias, which is subtracted from the sEMGdata-stream in order to remove the DC component of the sEMG signal. Additionally, this data is used to calculate the parameters $\mu_{\mathrm{ssc}}$ and $\mu_{\mathrm{zc}}$ for the respective features, such that these features show no activity within this data-set. This calculation is performed using a binary search to fulfill the following conditions:

$$
\begin{aligned}
& \forall i \in\{1, \ldots, 4850\}: R_{\mathrm{ssc}}\left(X_{\text {rest }}[i, \ldots, i+149]\right)=0 \\
& \forall i \in\{1, \ldots, 4850\}: R_{\mathrm{zc}}\left(X_{\text {rest }}[i, \ldots, i+149]\right)=0
\end{aligned}
$$

The combination of features can now efficiently be used to detect whether a user is resting or in an active state, by use of a threshold-crossing-method.

3) Recording of training data: To initialize the Gaussian Processes to decode the velocity commands, training data of the user's sEMG-activity needs to be recorded. Thus, the user is sequentially asked to provide sEMG-activity which he intends to be associated with motion along the cardinal axes (i.e. left, right, forward, backward, up and down). Within this process, the activity detection is used to track how much sEMG-data has been recorded for a desired direction, and notify the user to relax, as soon as enough data is recorded (typically 2 seconds of data per direction). In order to be able to separate and label the sEMG-data with the directions accordingly, the user is requested to relax for at least 1 second, after each directional activity.
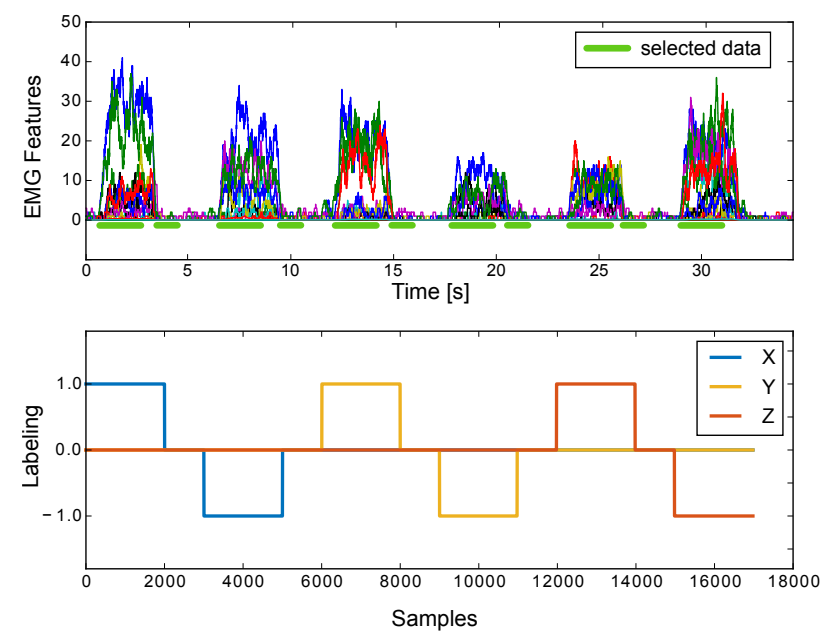

Fig. 3. Top: Exemplary training dataset and the data selected for labeling, based on the activity detection. Bottom: Generated labeling for the relevant data samples, based on the known sequence of directional activity.

Recording of one complete cycle of directional training data lasts approximately 35 seconds. To achieve variety in the user's sEMG signals, typically three repetitions of directional training are recorded.
4) Calculation of training dataset: From each training dataset, 2000 samples of each directional activity are selected for labeling based on the activity detection signal. Additionally, the 1000 samples of continuous resting activity, which follow each directional training, are also selected. The selected samples can easily be labeled according to the known sequence of directional activity. An exemplary plot of a single training data set and the created labeling can be seen in Figure 3. To achieve the three degree of freedom velocity decoding, three independent Gaussian Processes are used, each to model one degree of freedom, based on the generated training dataset.

5) Online regression: Once the Gaussian Processes are calibrated, they can be employed online, in order to decode the user commands from the sEMG-activity. Since independent Gaussian Process are used for the three single degrees of freedom, users can also produce combined commands. The online decoding process is depicted in Figure 4.

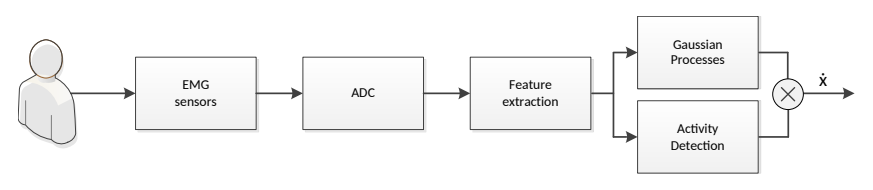

Fig. 4. Scheme of the EMG decoding

\section{ASSESSMENT}

To assess the performance of the sEMG-based interface, we tested it with two 49-year-old female subjects (S1 and S2) suffering from spinal muscular atrophy. Both subjects have also participated in [9] and therefore have previously used sEMG-based interfaces. However, the last time that they took part in such a study was 2.5 years prior to the experiments reported here. Additionally to the two subjects affected with muscular atrophy, data was also recorded from one subject (SR, age 27, female) with no known medical condition. Subject SR has regularly used the sEMG-based interface over the past two years in laboratory conditions for testing and evaluation. Therefore, SR can be considered as a very experienced user and data from this subject serves as a baseline. All subjects gave written consent to participate in the study, which was approved by the ethics commission of the faculty of medicine at the Technical University in Munich, Germany.

Assessment of the interface is composed of an offline evaluation of the EMG data and an online evaluation in which the subjects performed a reaching task based on Fitts-Law [11]. Offline evaluation is used to calculate the contribution of the individual electrodes and features to the calibration of the EMG-decoder. Online evaluation was performed to see how well the subjects could actually use the interface in a closed loop task.

Subjects S1 and S2 took part in the experiments in four sessions conducted on four consecutive days. Due to the severe muscular atrophy, electrode placement (see Figure 5) was performed based on visual inspection of the EMGsignals in combination with the feedback of the participants 

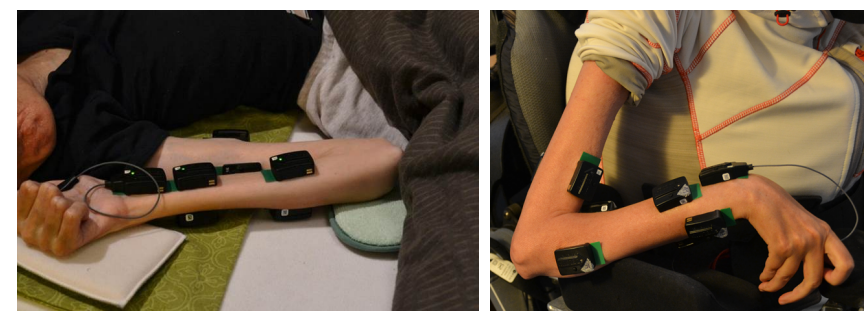

Fig. 5. Exemplary placement of the electrodes at the right arm of each participant. Eight surface EMG-sensors were used respectively. Left: participant S1; Right: participant S2.

TABLE I

LIST OF MUSCLES ANATOMICALLY CLOSEST TO RECORDING SITES

\begin{tabular}{|c|c|}
\hline \multicolumn{2}{|c|}{ Electrode placement } \\
\hline S1 & S2 \\
\hline thenar & thenar \\
hypothenar & flexor digitorum superficialis \\
flexor carpi radialis & extensor digiti minimi \\
extensor digiti minimi & flexor carpi radialis \\
brachioradialis & flexor carpi ulnaris \\
extensor carpi ulnaris & brachioradialis \\
triceps brachii & biceps brachii \\
pectoralis major & pectoralis major \\
\hline
\end{tabular}

about locations at which they were able to feel their remaining muscular activity. In fact, shifting electrode position by as much as $1 \mathrm{~cm}$ could result in complete loss of a viable EMGsignal. Placement of electrodes was manually optimized over the four experimental sessions. The final electrode placement is shown in Table I which lists muscles anatomically most closely related to the electrode placement, based on the physiology of the human arm. However, it has to be noted that due to the strong muscular atrophy, actual muscles that have been recorded may differ from this list.

Once the electrodes were placed, the decoder initialization was performed as described in Section II. After decodercalibration, online assessment was performed using a 2D and a 3D fitts-task. The 2D task was realized using a virtual environment displayed on a computer screen, while for the 3D task, a DLR light-weight robot was used. In the 2D task, subjects could control the velocity of a cursor moving in the virtual environment based on the velocity commands decoded from their EMG-signals. The virtual environment was designed to represent an area of $0.6 \mathrm{~m}$ by $0.6 \mathrm{~m}$ in which the cursor (circular shape with radius of $0.01 \mathrm{~m}$ ) could be moved with maximum velocity of $0.2 \mathrm{~m} / \mathrm{s}$. Circular targets with radius $(0.025 \mathrm{~m}$ and $0.0325 \mathrm{~m})$ were presented at a defined distance $(0.16 \mathrm{~m}, 0.24 \mathrm{~m}, 0.32 \mathrm{~m})$ to the current position of the cursor. Upon appearance of the target, subjects had to move the cursor within the target area. A target was counted as acquired, when the cursor was fully enclosed and had reached zero velocity. If the task was not accomplished after 22 seconds, it would be aborted and counted as timedout. The task was split in two parts, a training part in which 12 targets were presented in order to get acquainted with the interface and a testing part in which 36 targets had to be acquired, which was used for the actual assessment.

The six possible variations resulting from the two target- radii and the three target-distances, were pseudo-randomly permuted to achieve uniform distribution of these conditions. After the 2D task, a 3D fitts task using a DLR light weight robot was performed. Similar to the virtual environment, the reachable area of the robot was limited to a cube with edge length of $0.6 \mathrm{~m}$ and the endeffector-velocity of the robot was limited to $0.2 \mathrm{~m} / \mathrm{s}$. The target to be reached was made of a sensorized foam ball mounted on a telescopic rod. In this task, the target diameter was fixed and it was placed in the workspace of the robot at a randomly selected position, having a distance pseudo-randomly chosen from four available options $(0.16 \mathrm{~m} 0.24 \mathrm{~m} 0.32 \mathrm{~m} 0.4 \mathrm{~m})$. To acquire the target, subjects had to touch it with a spherical tool held by the robot, which was detected from an accelerometer build into the target. Similar to the 2D task, a task was counted as timed-out when not reaching a target within 22 seconds and subjects could get acquainted with the task in the first 12 trials, followed by 36 trials to be evaluated in the analysis. Figure 6 depicts one of the subjects while performing the 3D fitts-task with the robotic system.

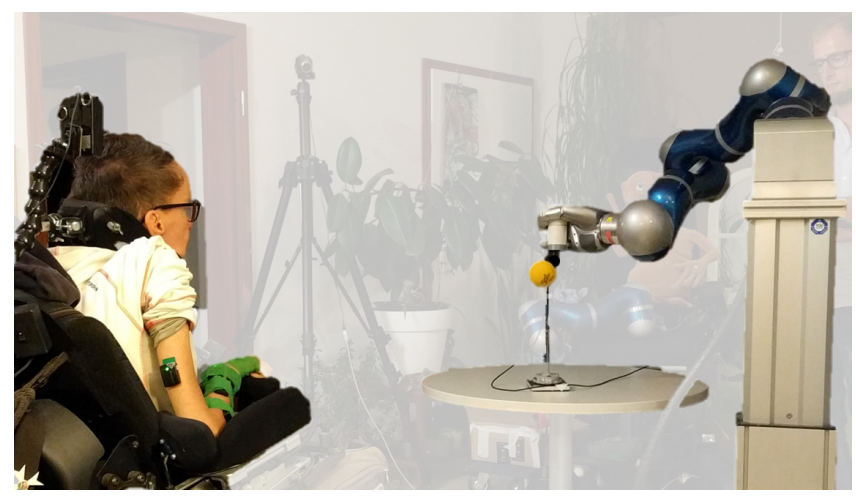

Fig. 6. S2 performing the fitts tasks with a 7 DoF light-weight robot. The translational velocity of the endeffector is commanded with the sEMG-based interface. The yellow ball depicts the sensorized target ball.

\section{Results}

To analyze the contribution of the single electrodes and the respective features, an offline analysis based on the data gathered during the training procedure was conducted. In this analysis, features were sequentially added for initialization of the Gaussian Process, based on their contribution to the decoding. I.e. In a first run, 32 (four feature-types times eight electrodes) Gaussian Processes were calculated each on one distinct feature-electrode-combination. For this calculation, two of the three available training data-sets were taken, while the third was used to calculate a root-mean-squared error to rate the performance and detect the feature with highest information content. In the next step, this "best feature" was combined with each of the remaining 31 features to find the second most relevant feature. This process was iteratively continued, until in the end, all features are rated according to their contribution.

Figure 7 (main plot) depicts the root mean squared error over the first 15 steps of this iterative process, using the 
training data of day 4 for subjects S1 and S2 and a reference data-set for SR. It is notable, that the information gain per added feature is much stronger for non-pathological subject SR compared to S1 and S2. The subplot in the top-right corner shows the occurrence of the individual feature classes, from which no preferred class of features can be identified. The iterative analysis also revealed, that information from each single electrode is used at the latest after adding 11 features to the decoding process (this information is not depicted in the plot).

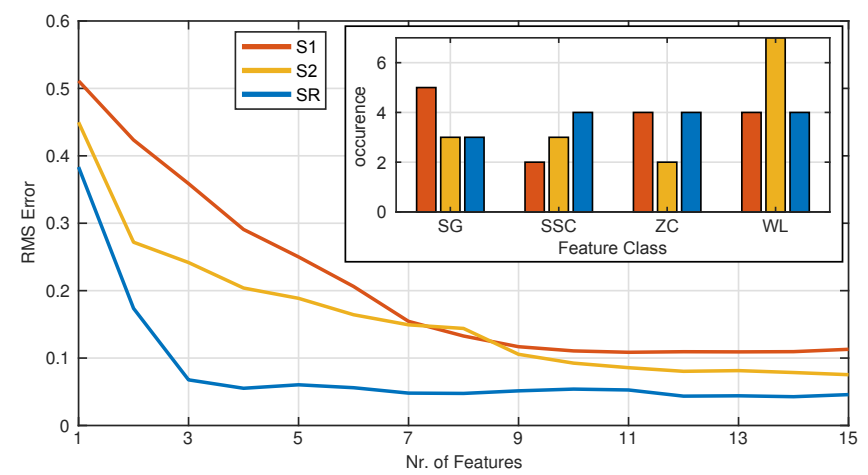

Fig. 7. Results of the feature contribution analysis when iteratively adding features to to the decoding process. Main plot: RMS-error with increasing number of features. Subplot: Number of occurrence per feature class among the 15 best features.

While this analysis suggest, that the selected features and the optimized electrode placement contribute well to the decoding of 3DoF velocity commands from sEMG, the more important aspect is, whether this approach also generalizes well during online decoding. Therefore, subjects S1 and S2 performed a reaching task based on Fitts' Law in each of the four experimental sessions. In the field of human-computer interaction, Fitts' Law is widely used in order to compare information transfer in human reaching tasks [?]. According to Fitts' Law, the movement time (MT) needed for a reaching motion is directly proportional to the Index of Difficulty (ID), which is computed according to Eq. (6), where D is the distance to the target, and $\mathrm{W}$ if the width of the target along the direction of motion.

$$
\mathrm{ID}=\log _{2}\left(\frac{\mathrm{D}}{\mathrm{W}}+1\right)
$$

In this study, reaching tasks were either performed using a cursor moving in a virtual environment, or a robotic hand-arm system. Having multiple repetitions of reaching tasks, with different IDs, one can calculate the regression line as depicted in Figure 8. From the figure, it is notable, that performance of subject S1 increased on day 3 and 4 compared to the first two days. Furthermore, it is notable, that movement times of the experienced user SR are shorter than for subject S1. For numerical comparison of the performance in a Fitts' task, one can calculate the Index of Performance (IP) as:

$$
\mathrm{IP}=\frac{1}{x} \sum_{i=1}^{x} \frac{\mathrm{ID}_{\mathrm{i}}}{\mathrm{MT}_{\mathrm{i}}}
$$

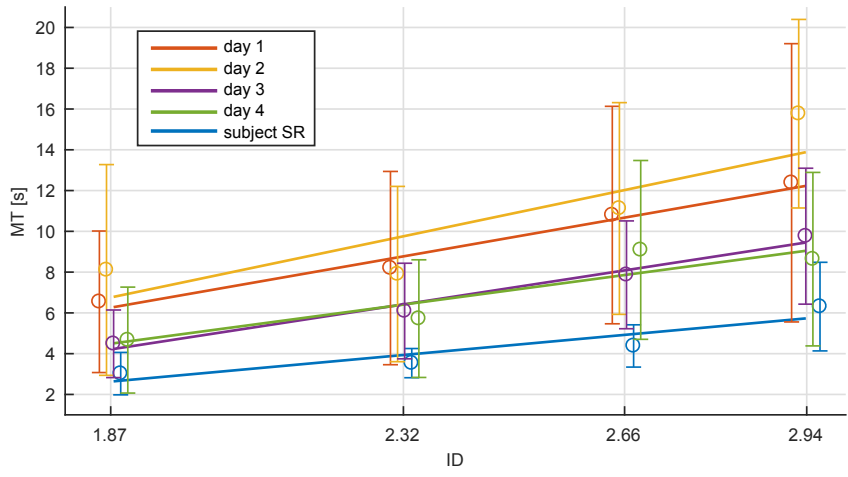

Fig. 8. Results for the three-dimensional Fitts' task over four days for subject $\mathrm{S} 1$ in comparison to the non-pathological subject SR.

TABLE II

INDEX OF PERFORMANCE AND NUMBER OF TIMEOUTS FOR THE 2D AND 3D FITTS' TASK.

\begin{tabular}{|c|c|c|c|c|c|}
\hline \multicolumn{2}{|c|}{} & \multicolumn{2}{|c|}{ 2D } & \multicolumn{2}{c|}{ 3D } \\
\hline Subject & Day & IP & \#TO & IP & \#TO \\
\hline \hline S1 & 1 & 0.573 & 0 & 0.366 & 10 \\
\hline S1 & 2 & 0.606 & 0 & 0.324 & 2 \\
\hline S1 & 3 & 0.460 & 2 & 0.403 & 0 \\
\hline S1 & 4 & 0.928 & 0 & 0.453 & 2 \\
\hline \hline S2 & 1 & 0.814 & 0 & 0.481 & 0 \\
\hline S2 & 2 & 0.715 & 0 & 0.491 & 0 \\
\hline S2 & 3 & 0.966 & 0 & 0.569 & 0 \\
\hline S2 & 4 & 0.995 & 0 & 0.605 & 0 \\
\hline \hline SR & - & 1.154 & 0 & 0.630 & 0 \\
\hline
\end{tabular}

Table II lists the index of performance and the number of timed-out reaching tasks (\#TO) for the 2D and 3D tasks for all subjects. An improvement in performance is evident.

\section{Discussion}

The goal of this study was to design an sEMG-based interface which allows people with severe muscular atrophy to continuously control assistive devices in three degrees of freedom. To achieve this, several improvements have been made to the interface design as used in the proof of concept study [9]. To verify the improvement, reach and grasp tasks have been performed and analyzed. The offline analysis of the information contribution suggests that all feature classes used in the decoding process provide information in the decoding. As one would expect from the physiology of the sEMG signal of atrophic muscles, a higher number of features/electrodes is needed in the decoding process as compared to non-pathological EMG-signals.

The results of the 2D and 3D Fitts' task show that the performance of subjects S1 and S2 gets close to that of the reference subject SR, even though the amount of information acquired from atrophic muscles is lower than in regular EMG. Additionally, it has to be noted, that subject SR has a very long experience in using the sEMG-based interface to control movement of a robotic arm ( $>50$ hours of net usage). Based on this, it would be very interesting to see, whether subjects $\mathrm{S} 1$ and S2, as well as other subjects with muscular atrophy are able to achieve comparable performance after 
long term training.

In comparison to the original approach of [9], it is evident, that the performance could be considerably improved. The task performed in the original approach was comparable to the 3D Fitts' task, except that target distance was constantly kept at $0.3 \mathrm{~m}$. Average reaching times in the original approach were approximately 16, 40, 37 and 23 seconds, which is considerably higher than the average times for the two longest distances in the results of this study $(<16$ seconds in all sessions).

The results of this study suggest, that the devised sEMG based interface can be used by people with atrophic muscles to move a robotic manipulator in three degrees of freedom. Additional studies have to be conducted to analyze, whether control achieved with this interface is precise enough to accomplish functional tasks in combination with a robotic system. Furthermore, the interface needs to be validated with a larger group of users as well as users with muscular atrophy resulting from other medical conditions.

\section{REFERENCES}

[1] R. L. Bennett, "The evolution of the georgia warm springs foundation feeder," Physical Therapy Review, vol. 36, no. 11, 1956.

[2] G. Kramer, G. Römer, and H. Stuyt, "Design of a dynamic arm support (das) for gravity compensation," 2007, pp. 1042-1048.

[3] V. Maheu, P. S. Archambault, J. Frappier, and F. Routhier, "Evaluation of the jaco robotic arm: Clinico-economic study for powered wheelchair users with upper-extremity disabilities," in Rehabilitation
Robotics (ICORR), 2011 IEEE International Conference on. IEEE, 2011, pp. 1-5.

[4] H. H. Kwee, "Integrated control of manus manipulator and wheelchair enhanced by environmental docking," Robotica, vol. 16, no. 5, pp. 491-498, 1998.

[5] L. R. Hochberg, D. Bacher, B. Jarosiewicz, N. Y. Masse, J. D. Simeral, J. Vogel, S. Haddadin, J. Liu, S. S. Cash, P. van der Smagt et al., "Reach and grasp by people with tetraplegia using a neurally controlled robotic arm," Nature, vol. 485, no. 7398, p. 372, 2012.

[6] J. L. Collinger, B. Wodlinger, J. E. Downey, W. Wang, E. C. TylerKabara, D. J. Weber, A. J. McMorland, M. Velliste, M. L. Boninger, and A. B. Schwartz, "High-performance neuroprosthetic control by an individual with tetraplegia," The Lancet, vol. 381, no. 9866, pp. 557-564, 2013

[7] F. Nijboer, E. Sellers, J. Mellinger, M. A. Jordan, T. Matuz, A. Furdea, S. Halder, U. Mochty, D. Krusienski, T. Vaughan et al., "A p300based brain-computer interface for people with amyotrophic lateral sclerosis," Clinical neurophysiology, vol. 119, no. 8, pp. 1909-1916, 2008.

[8] S. M. Grigorescu, T. Lüth, C. Fragkopoulos, M. Cyriacks, and A. Gräser, "A bci-controlled robotic assistant for quadriplegic people in domestic and professional life," Robotica, vol. 30, no. 3, pp. 419431, 2012.

[9] J. Vogel, J. Bayer, and P. Van Der Smagt, "Continuous robot control using surface electromyography of atrophic muscles," in Intelligent Robots and Systems (IROS), 2013 IEEE/RSJ International Conference on. IEEE, 2013, pp. 845-850.

[10] K. Veer and T. Sharma, "A novel feature extraction for robust emg pattern recognition," Journal of medical engineering \& technology, vol. 40, no. 4, pp. 149-154, 2016.

[11] P. M. Fitts, "The information capacity of the human motor system in controlling the amplitude of movement." Journal of experimental psychology, vol. 47, no. 6, p. 381, 1954. 